\title{
Role of cell therapy in Parkinson disease
}

\author{
Olle Lindvall, M.D., Ph.D., And Peter Hagell, R.N., Ph.D. \\ Section of Restorative Neurology, Wallenberg Neuroscience Center, University Hospital, Lund, \\ Sweden
}

\begin{abstract}
Clinical studies involving intrastriatal transplantation of embryonic mesencephalic tissue in patients with Parkinson disease (PD) have provided proof-of-principle for the cell replacement strategy in this disorder. The grafted dopaminergic neurons can reinnervate the denervated striatum, restore regulated dopamine release and movement-related frontal cortical activation, and produce significant symptomatic relief. In the most successful cases, patients have been able to withdraw from levodopa treatment after undergoing transplantation and resume an independent life. There are, however, several problems linked to the use of primary embryonic tissue: 1) lack of sufficient amounts of tissue for transplantation in a large number of patients; 2) variability of functional outcome (major improvement in some and modest if any clinical benefit in others); and 3) occurrence of troublesome dyskinesias in a significant proportion of patients after transplantation. Thus, neural transplantation is still at an experimental stage in the treatment of PD. For the development of a clinically useful cell therapy we need to define better criteria for patient selection and how graft placement should be optimized in each individual. Most importantly, we need to generate large numbers of viable dopamine neurons in preparations that are standardized and quality controlled. Stem cells could be useful as an unlimited source of dopamine neurons. Thus far, neurons with at least some dopaminergic characteristics have been generated from stem cells. In most cases, however, their survival after grafting in animals has been poor, and it is also unclear if they function as normal dopamine neurons. Several scientific issues need to be addressed before stem cell-based therapies can be tested in PD patients.
\end{abstract}

\section{Key Words - Parkinson disease • neural transplantation • stem cell • striatum positron emission tomography $\bullet$ dopamine}

The cell replacement strategy in PD has been based on the idea that neural graft-induced restoration of dopamine neurotransmission in the striatum, even if the disease is chronic and also affects other neuronal systems and brain regions, could lead to substantial and long-lasting functional recovery. It was demonstrated more than 20 years ago that embryonic mesencephalic dopamine-rich tissue implanted in a rat model of PD reinnervated the denervated striatum and ameliorated some functional deficits. Extensive animal studies subsequently showed that the grafted dopamine neurons display many of the morphological and functional characteristics of intrinsic dopamine neurons; that is, they reinnervate the denervated striatum and form synaptic contacts with host neurons, are spontaneously active and release dopamine, and receive afferent input from the host. ${ }^{3}$ The graft-induced reinnervation is accompanied by significant amelioration of several but not all aspects of the dopamine deficiency syndrome both in rodents and monkeys. ${ }^{1,3}$

Abbreviations used in this paper: $\mathrm{bFGF}=$ basic fibroblast growth factor; DLPFC $=$ dorso-lateral prefrontal cortex; $\mathrm{PD}=$ Parkinson disease PET = positron emission tomography; $\mathrm{rCBF}=$ regional cerebral blood flow; SMA = supplementary motor area; $\mathrm{TH}=$ tyrosine hydroxylase; UPDRS = United PD Rating Scale.
Clinical trials involving transplantation of human embryonic mesencephalic tissue to the striatum in patients with PD were initiated in 1987. At that time the efficacy of cell replacement in the diseased human brain was unknown. In many of the scientific efforts during the past 15 years the main objective has been to provide proof-ofprinciple that: 1) the grafted dopamine neurons can survive and form connections in the brains of PD patients; 2) the patient's brain can integrate and use the grafted neurons; and 3) the grafts can induce a measurable clinical improvement. Most clinical studies have been performed as open-label trials in relatively small groups of patients. Two double-blinded randomized clinical trials, however, were initiated in the mid-1990s to evaluate the effectiveness of neural grafting according to current procedures compared with that of sham surgery.

In this article, we will first argue, based on the clinical experiences with neural transplantation thus far, that cell replacement therapy can be effective in patients with PD. We will also conclude, however, that the use of primary embryonic tissue is associated with problems related to availability, standardization, variation in patient-related functional outcome, and adverse effects. In light of these factors, we will then discuss the possible role of the stem cell technology for the further development of a cell therapy in the treatment of PD. 


\section{CLINICAL EXPERIENCES WITH NEURAL TRANSPLANTS IN PD}

\section{Short- and Long-term Graft Survival and Growth}

To date, approximately 350 patients with PD have undergone grafting procedures involving primary embryonic tissue of human or porcine origin. It is well established that human embryonic mesencephalic dopamine neurons can survive transplantation into the brain of PD patients. Significant increase of $\left[{ }^{18} \mathrm{~F}\right]$ fluorodopa uptake in the grafted striatum has been shown using PET in more than 40 patients with PD. . $^{5,11,14,15,23,25,28,29,33,34,39}$ In one patient, the fluorodopa uptake in the putamen was normalized after transplantation. ${ }^{30,39}$ Investigators in histopathological studies have confirmed the survival of dopaminergic grafts and demonstrated reinnervation of the striatum in two parkinsonian patients who died after transplantation. ${ }^{18-20}$ In these two patients, between 80,000 and 135,000 dopaminergic neurons had survived on each side, with neuritic outgrowth from the grafted neurons extending up to approximately $7 \mathrm{~mm}$ within the putamen. With six tracts, placed $5 \mathrm{~mm}$ apart, confluent reinnervation of 24 to $78 \%$ of the designated target area in the postcommissural putamen could be obtained, although in the patient with the densest reinnervation the putamen was shrunken. ${ }^{20}$ The dopaminergic innervation occurred in a patch-matrix pattern and electron microscopy showed synaptic connections between graft and host. There was no evidence that sprouting had occurred in the patients' own dopamine neurons.

Grafts of human mesencephalic tissue can exhibit longterm survival despite an ongoing disease process and continuous antiparkinsonian drug treatment. In two patients, in whom grafts were placed unilaterally in the putamen, the fluorodopa uptake in the grafted structure was still high at 6 and 10 years after surgery, respectively. ${ }^{30,39} \mathrm{In}$ contrast, there had been a progressive decrease of tracer uptake in nongrafted striatal regions, indicating degeneration of the patients' own dopamine neurons. Immunological rejection of the grafts has not been reported in PD patients, even several years after withdrawal of immunosuppression therapy.

\section{Magnitude of Clinical Improvement}

Several clinical research groups have demonstrated therapeutic improvement associated with graft survival. ${ }^{5,8,11,14,15,23,25,26,28,29,33,34,39}$ In the most successful cases, levodopa treatment was withdrawn during several years after transplantation. ${ }^{5,14,39}$ For a more detailed account of clinical observations, including constraints and morbidity, following neural transplantation in PD, refer to two previous studies. ${ }^{22,24}$

Table 1 provides a summary of the magnitude of the overall clinical benefit at 10 to 24 months postoperatively in four series of patients in whom human embryonic mesencephalic tissue grafts were placed bilaterally. In the three open-label trials, ${ }^{5,14,15}$ patients underwent graft implantation bilaterally with primary human embryonic mesencephalic tissue, obtained from approximately three to five donors, into each putamen. In some cases, tissue was also implanted in the caudate nucleus. According to the $\mathrm{UPDRS}^{9}$ motor score obtained during a practically de- fined off-medication period (that is, in the morning, at least 12 hours after the last dose of antiparkinsonian medication), the overall symptomatic relief at 10 to 24 months postoperatively was between 30 and $40 \%$. In addition, there was a decrease (43-59\%) of the mean daily time spent in the off-medication phase. The mean daily levodopa requirements were reduced by 16 to $45 \% .^{5,14,15}$ It is interesting to note that in these three studies, even if increased fluorodopa uptake was demonstrated (by 60\%) in the putamen, indicating graft survival, the uptake after transplantation was still only approximately $50 \%$ of the normal mean. This finding probably explains, at least to some extent, the incomplete functional recovery and indicates that there is room for considerable improvement.

In the only double-blind, placebo-controlled study reported to date ${ }^{10}$ investigators demonstrated a more modest clinical response, with $18 \%$ reduction of UPDRS motor score in off-medication periods at 12 months after placement of bilateral putaminal grafts but no improvement in the sham-operated group. In patients younger than 60 years of age, the improvement of UPDRS scores was $34 \%$. These data are important because they provide the first direct evidence of a specific graft-induced improvement, distinguishable from a placebo effect. In this trial, less tissue was implanted compared with that in the openlabel trials and, in agreement, the increase of fluorodopa uptake was lower (only $40 \%$ compared with $60 \%$, respectively). In two patients who died after graft implantation, the numbers of dopaminergic neurons in each putamen were only between 7000 and $40,000,{ }^{10}$ whereas in the two patients in one of the open-label trials the dopaminergic cell counts ranged from 80,000 to $135,000 .^{18-20}$ The low cell number is probably due to the fact that tissue obtained in only two donors was implanted in each putamen (compared with tissue obtained in three to five donors in the open-label trials) and that the tissue was stored in cell culture for up to 4 weeks before implantation. In agreement, the postoperative clinical improvement was smaller than that in the other patient series. These findings provide further support for the notion that the number of viable implanted dopamine neurons is an important factor determining the magnitude of symptomatic relief. ${ }^{12}$

\section{Dyskinesias After Neural Transplantation}

Severe dyskinesias during the off-medication phases were observed by Freed, et al., ${ }^{10}$ in $15 \%$ of their patients after neural transplantation. In a comprehensive, retrospective analysis of 14 patients followed for up to 11 years after grafting, Hagell, et al. ${ }^{13}$ found that hyperkinesias (predominantly choreoid movements) and dystonias increased during off-medication phases postoperatively, whereas there were no statistically significant changes in the severity of peak on-medication-phase dyskinesias. In eight patients, dyskinesias were mild and caused no distress or disability; in the remaining six, dyskinesias were of moderate severity and constituted in one patient, a clinical therapeutic problem. Freed, et al. ${ }^{10}$ proposed that the dyskinesias were due to a continued fiber outgrowth from the graft resulting in a relative dopamine excess. In patients assessed by Hagell, et al., ${ }^{13}$ however, the severity of dyskinesias was not related to the magnitude of graftderived dopaminergic reinnervation or symptomatic relief 
Table 1

Summary of overall clinical benefit in four series of patients treated with bilateral intrastriatal implantation of embryonic mesencephalic tissue*

\begin{tabular}{|c|c|c|c|c|c|c|c|}
\hline \multirow{2}{*}{$\begin{array}{l}\text { Authors \& Year } \\
\text { (No. of Cases) }\end{array}$} & \multirow{2}{*}{$\begin{array}{c}\text { No. of } \\
\text { VM/Putamen }\end{array}$} & \multicolumn{3}{|c|}{ Putamen Fluorodopa Uptake (\%) } & \multirow{2}{*}{$\begin{array}{c}\text { UPDRS } \\
\text { Score Off } \\
\text { Medication }(\%) \ddagger\end{array}$} & \multirow{2}{*}{$\begin{array}{c}\text { Daily } \\
\text { Time in Off } \\
\text { Phase }(\%)\end{array}$} & \multirow{2}{*}{$\begin{array}{c}\text { Daily } \\
\text { Dose of } \\
\text { Levodopa }(\%)\end{array}$} \\
\hline & & Preop $\dagger$ & Postop $\dagger$ & Change & & & \\
\hline Hagell, et al., 1999 (4)§ & 4.9 & 31 & 52 & +69 & -30 & -59 & -37 \\
\hline Hauser, et al., 1999 (6) & $3-4$ & 34 & 55 & +61 & -30 & -43 & -16 \\
\hline Brundin, et al., 2000 (5) & $2.8 \|$ & 31 & 48 & +55 & -40 & -43 & -45 \\
\hline Freed, et al., 2001 (19) & 2 & NR & NR & +40 & -18 & NR & NR \\
\hline
\end{tabular}

$* \mathrm{NR}=$ not reported; $\mathrm{VM}=$ ventral mesencephalon.

$\dagger$ Mean percentage of uptake compared with normal mean determined in healthy volunteers.

\$ As assessed during the practically defined off-medication phase.

$\S$ Excludes one patient with possible multisystem atrophy.

॥ The graft tissue was treated with lazaroid tirilazad mesylate.

(Fig. 1). Thus, these data do not provide any evidence of an underlying dopaminergic overgrowth, nor that effective dopamine neuron replacement and major recovery of motor function occur in conjunction with the development of severe dyskinesias. The development of severe dyskinesias is not a characteristic feature of dopamine cell replacement per se and therefore should not stop the further development of a cell therapy for PD. The underlying mechanisms must be better understood, however, so that off-medication-phase dyskinesias following neural transplantation can be avoided.

\section{Mechanisms of Graft Function}

The aforementioned clinical observations demonstrate that the grafts can survive, store dopamine, and produce symptomatic relief in PD patients. The authors of recent studies also provide evidence that grafts of primary human embryonic mesencephalic tissue can restore regulated release of dopamine in the striatum and that grafts can become functionally integrated into the neural circuitries in the patient's brain.

One patient in whom a graft was unilaterally transplanted in the putamen has experienced significant clinical improvement and, in agreement, fluorodopa uptake in the grafted putamen was normalized at 3 years with no further change at 10 years postoperatively (Fig. 2).$^{30} \mathrm{In}$ contrast, the putamen in which no graft was implanted exhibited a progressive decrease of fluorodopa uptake, which at 10 years postoperatively was approximately $10 \%$ of the normal level. Dopamine release was quantified at 10 years by using $\left[{ }^{11} \mathrm{C}\right]$ raclopride and PET to measure dopamine D2 receptor occupancy by endogenous dopamine. ${ }^{30}$ Both basal- and amphetamine-induced dopamine release was normal in the grafted putamen, whereas the release in the contralateral nongrafted putamen was very low (Fig. 2). It seems highly likely that the efficient restoration of dopamine release in large parts of the grafted putamen underlies the patient's major clinical improvement.

In the one study the activation of two frontal cortical areas associated with movements, the SMA and the DLPFC, was analyzed using rCBF measurement with PET in four patients in whom grafts were implanted bilaterally in the caudate and putamen. ${ }^{31}$ The SMA and DLPFC are known to be important in the preparation and selection of voluntary movements, their function is influenced by the basal gangliathalamocortical neural circuitries, and their impaired activation is believed to underlie parkinsonian akinesia. Preoperatively, investigators

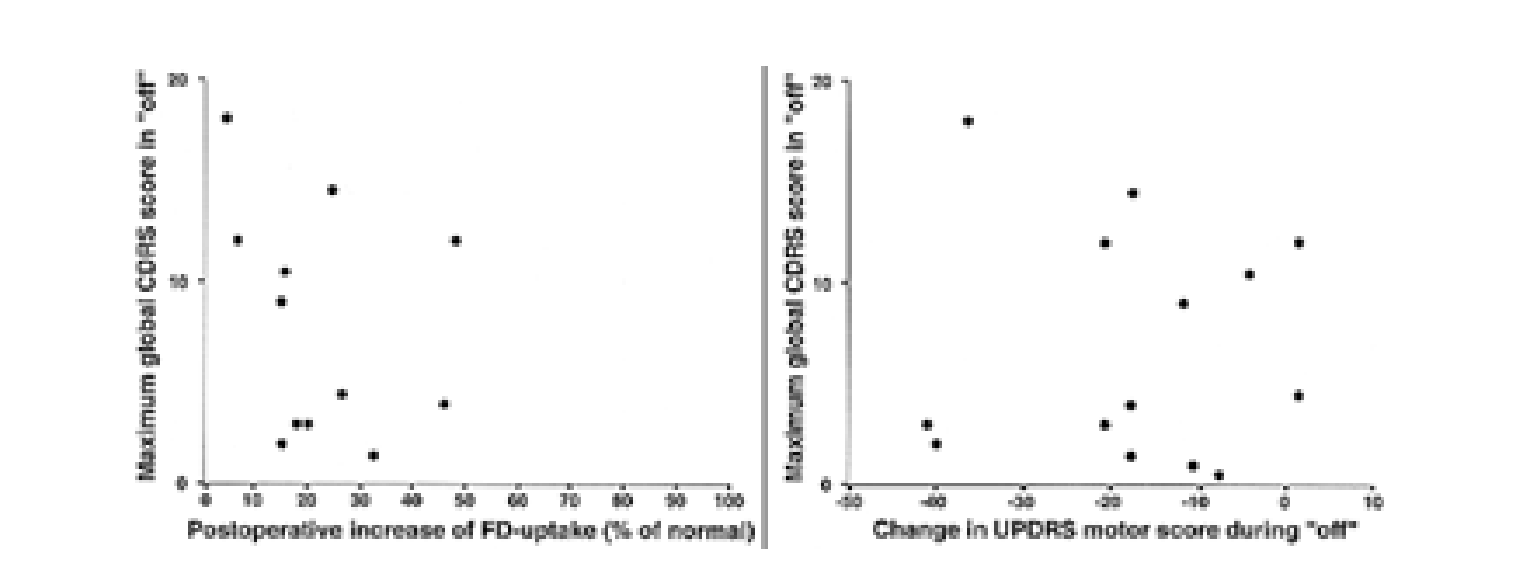

Fig. 1. Off-medication phase dyskinesias following neural transplantation are not linked to effective dopamine cell replacement and major clinical improvement. Scatterplots of the relationship between the global Clinical Dyskinesia Rating Scale (CDRS) scores in practically defined off-medication and the change in fluorodopa uptake, expressed as percent of normal mean (left), and in UPDRS motor score (right) determined at the time of maximum postoperative offmedication-phase CDRS scores. Used with permission from Hagell, et al., 2002. 


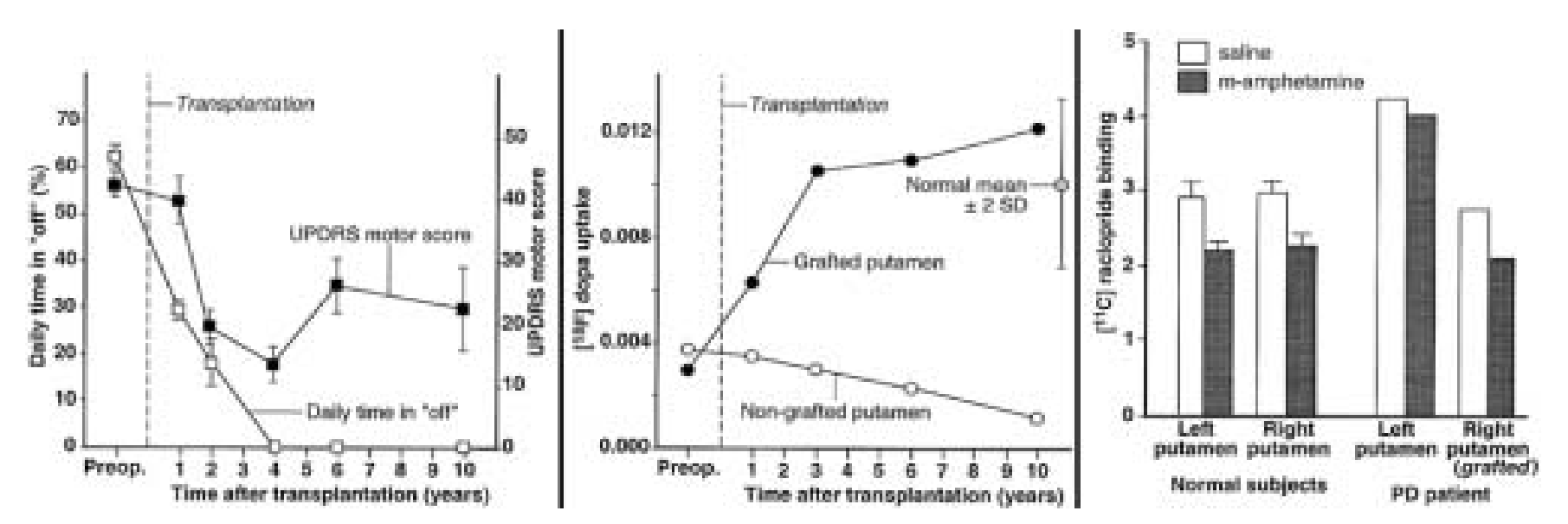

Fig. 2. Neural grafts can restore dopamine storage and release in the striatum to normal levels, yielding major symptomatic relief for more than a decade in patients with PD. A: Percentage of the day spent in the off-medication phase (open squares) and UPDRS motor score (solid squares) in the practically defined off-medication phase preoperatively and at various time points after transplantation of human embryonic mesencephalic tissue unilaterally into the right putamen. Data are presented as mean $\pm 95 \%$ confidence interval. Dashed vertical line indicates time of transplantation. B: Fluorodopa uptake in the grafted (solid circles) and nongrafted (open circles) putamen in the same patient. Comparative data (mean \pm 2 standard deviation) are given for a group of 16 healthy volunteers (shaded circle with error bars). Dashed vertical line indicates time of transplantation. $\quad \mathrm{C}$ : Basal and drug-induced dopamine release as assessed using $\left[{ }^{11} \mathrm{C}\right] \mathrm{raclo}-$ pride PET to measure dopamine D2 receptor occupancy by the endogenous transmitter. In the baseline condition (saline infusion [open bars]), $\left[{ }^{11} \mathrm{C}\right]$ raclopride binding is increased in the nongrafted putamen in the patient, whereas it is normal on the grafted side (right putamen). After $\mathrm{m}$-amphetamine administration (solid bars), the binding reduction in the grafted putamen is similar to that seen in the putamen assessed in healthy volunteers, whereas it is negligible in the nongrafted putamen. Used with permission from Piccini, et al., 1999.

observed only a small activation of the SMA and no significant activation of the DLPFC (Fig. 3). No significant differences in activation were demonstrated in these patients at 6.5 months after graft implantation compared with preoperatively, whereas at 18.3 months there was significantly increased activation of both the SMA and DLPFC. The time course of clinical improvement paralleled that of the increase of cortical activation with partial recovery after 6.5 months and substantial improvement at 18.3 months (Fig. 3). In contrast, striatal fluorodopa uptake was already significantly elevated at 6.5 months, and no further change by 18.3 months was noted (Fig. 3). Taken together, these findings indicate that successful grafts in patients with PD, by improving striatal dopaminergic neurotransmission, can restore movement-related cortical activation, which probably is necessary to induce substantial clinical improvement. These data also provide new evidence that the functional effects of the grafted neurons go beyond those of a simple dopamine delivery system. Restoration of nonregulated dopamine release, as in the early stages of graft maturation when fluorodopa uptake is already significantly elevated, seems to be insufficient to improve cortical activation during movement and to induce maximum clinical recovery. To increase basal gangliathalamocortical neurotransmission and movement-related cortical activation, the grafted dopamine neurons likely need to establish both efferent and afferent synaptic connections with the host brain.

\section{Strategies to Increase Survival of Grafted Dopamine Neurons}

A major problem with current transplantation procedures is that the survival of grafted dopamine neurons is low, only 5 to $20 \%$, and therefore tissue obtained in six to eight donors is needed for each PD patient to induce significant clinical improvement. In animal experiments, the survival of grafted mesencephalic dopamine neurons can be increased two- to fourfold by exposure of the graft to growth factors, as well as compounds that reduce oxidative stress or inhibit caspases (for review, see the study by Brundin, et al. ${ }^{4}$ ). The only compounds that have been tested clinically are the lazaroid, tirilazad mesylate, and glial cell line-derived neurotrophic factor. ${ }^{5,28}$ The authors of these clinical studies have provided tentative evidence that both tirilazad mesylate and glial cell-derived neurotrophic factor (administered to the graft tissue during a 6-day pregrafting storage) may improve survival of grafted dopamine neurons in PD patients.

\section{Xenografts in PD}

In the initial attempts involving implantation of porcine xenografts in PD patients, the survival of dopamine neurons was poor and the clinical benefits questionable. ${ }^{7,35}$ Major concerns related to porcine xenografts, apart from immunological rejection and transfer of virus, are, first, that a very large number of porcine donors and many implant sites may be needed to reinnervate the human striatum effectively and, second, that the porcine dopamine neurons may have a lesser capacity to integrate functionally into the patient's brain compared with human dopamine neurons.

\section{FACTORS FOR A SUCCESSFUL CELL THERAPY IN PD}

For the further development of cell replacement thera- 


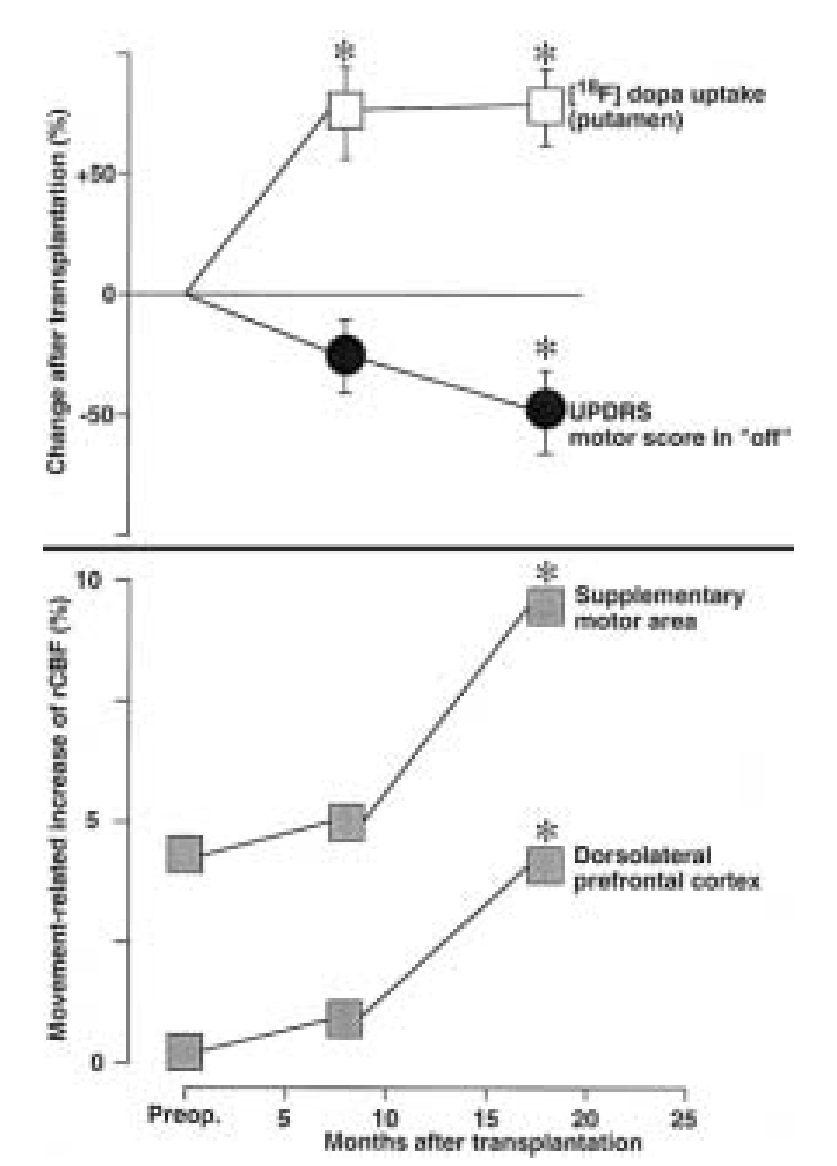

Fig. 3. Evidence for functional integration of dopaminergic grafts in PD patients. Changes of fluorodopa uptake in the putamen and UPDRS motor score in the off-medication phase (upper panel) and movement-related increases of regional cerebral blood flow compared with resting condition in the SMA and DLPFC (lower panel), preoperatively and at 6.5 and 18.3 months after bilateral implantation of human embryonic mesencephalic tissue into the putamen and caudate nucleus in four PD patients. Putaminal fluorodopa uptake is significantly elevated already at 6.5 months after transplantation with no further changes thereafter. In contrast, the symptomatic relief is only partial at 6.5 months and substantial clinical improvement, as measured by the UPDRS motor score, does not occur until the 2 nd postoperative year. The gradual and delayed symptomatic relief is paralleled by the recovery of movement-related cortical activation. Data are presented as mean \pm standard deviation. $* \mathrm{p}<0.001$, compared to preoperatively, t-test. Modified from Piccini, et al., 1999.

py in PD, it is of fundamental importance to determine what factors determine the magnitude of symptomatic relief in patients after transplantation. Based on the results obtained from the clinical trials as well as from studies in animal models, a set of graft-related requirements can be identified that must be fulfilled to induce marked and clinically valuable improvement. 1) The grafted cells have to express the complete cellular machinery for dopamine synthesis and release, and they must possess the properties of fully mature mesencephalic dopamine neurons, both morphologically and electrophysiologically. It should be pointed out, however, that we need to know also the role of nondopaminergic cells in the graft for the outcome after transplantation. These cells, which constitute approximately $90 \%$ of all cells in the primary embryonic mesencephalic graft, could contribute to the functional recovery but may also, hypothetically, induce or contribute to adverse effects such as dyskinesias. 2) At least approximately 100,000 grafted dopamine neurons should survive long term in each putamen. 3) The grafted dopamine neurons should reestablish a dense, functional, dopamine-releasing terminal network in large parts of the striatum. 4) The grafts have to become functionally integrated into host basal gangliathalamocortical circuitries. In the ideal scenario, the grafted neurons would be able to reconstruct the nigrostriatal pathway with appropriate afferent and efferent connections. 5) When tested preclinically in animal models of PD, the cells must be functional not only in tests of drug-induced behavior but also in tests of spontaneous motor behavior (akinesia and limb-use tests).

A successful cell therapy for PD, however, will also necessitate improved criteria and standards for patient selection and assessment as well as knowledge concerning optimal graft placement and dosage. The extent, degree, and rate of degeneration of dopaminergic and nondopaminergic neurons in the patient's brain will certainly influence to what extent a dopaminergic graft can restore normal function. If there is extensive dopaminergic denervation outside the grafted area or if other systems are severely affected, the degree of symptomatic relief is likely to be only modest compared with that in a patient in whom there is a more restricted dopaminergic denervation in the striatum, which has been successfully reinnervated by the graft. Furthermore, a rapid degeneration of the patient's own neuronal systems within and outside the grafted areas may only yield transient postoperative improvements. Detailed preoperative imaging modalities such as high-resolution fluorodopa-PET, will be invaluable tools to design the optimal transplantation procedure for each patient.

\section{STEM CELLS FOR CELL THERAPY IN PD}

There are two principally different ways of using stem cells for grafting in PD. First, the cells are predifferentiated in vitro to dopaminergic neurons prior to transplantation. Thus, stem cells could become an almost unlimited source for the generation of dopamine neurons. The cell preparations could be standardized and quality controlled with respect to viability and purity. Second, the progenitors differentiate in vivo to dopaminergic neurons after implantation into the striatum or substantia nigra. These neurons may integrate better compared with primary embryonic neurons and, in the ideal scenario, reconstruct the nigrostriatal pathway. Whether this will be possible, however, is at present unknown. It will require that the mechanisms to instruct the immature progenitors to differentiate into the missing dopamine neurons function also in the PD patient's brain. Some support for this strategy was provided in a recent report ${ }^{2}$ in which investigators found that undifferentiated mouse embryonic stem cells, implanted in low numbers into the dopamine denervated rat striatum, proliferated and that a proportion of them differentiated into cells expressing several markers of mesencephalic dopaminergic neurons. The grafts ameliorated drug-induced rotational asymmetry, but their capacity to reinner- 


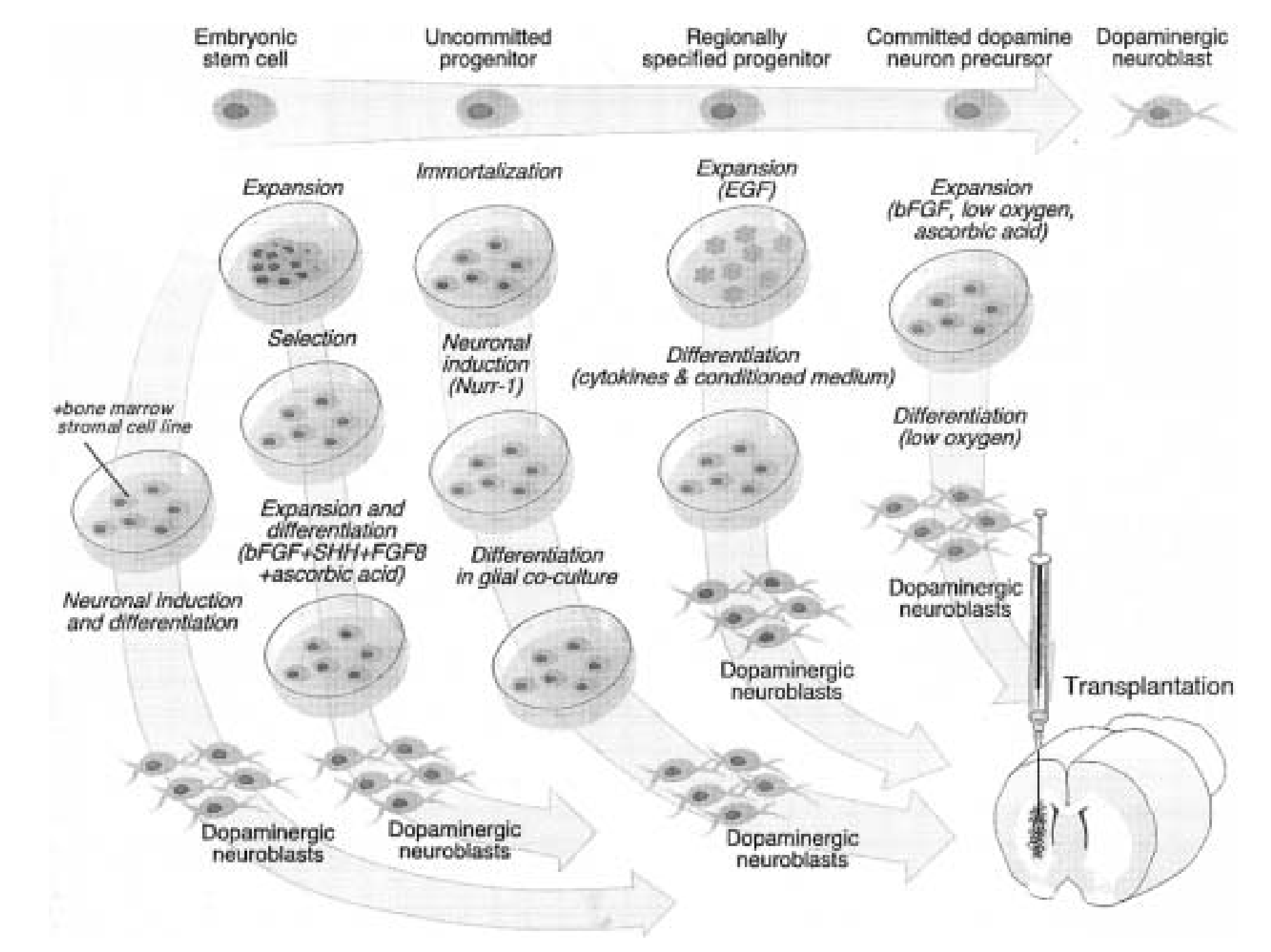

Fig. 4. Large numbers of cells with characteristics of dopamine neurons can be generated from in vitro expanded progenitors. The use of stem cells and immature progenitors for grafting in PD probably necessitates predifferentiation of the cells into dopaminergic neurons prior to implantation. This strategy has been explored using mouse embryonic stem cells, genetically engineered immortalized mouse neural stem cells, in vitro expanded regionally specified rat mesencephalic progenitors, and committed mesencephalic dopamine neuron precursors obtained from rat embryos. EGF $=$ epidermal growth factor; $\mathrm{SHH}=$ sonic hedgehog

vate the striatum and release dopamine, as well as to improve behavioral deficits resembling the symptoms in $\mathrm{PD}$, is unclear.

Hypothetically, dopamine neurons could be made from stem cells of the following four different sources: embryonic stem cells from the fertilized egg, neural stem cells from the embryonic or adult brain, or from stem cells in other tissues. The crucial question, however, is if those neurons will become functional dopamine neurons, fulfilling the aforementioned criteria. Another unresolved issue is whether nondopaminergic neurons and glial cells normally present in the primary mesencephalic grafts that have been placed in PD patients are important for the differentiation and function of the dopamine neurons. If this is the case, an enriched population of predifferentiated dopamine neurons may not be the optimal preparation.

The possibility to generate dopamine neurons for trans- plantation from stem cells or neuronal precursors has been explored by undertaking several approaches. Figure 4 provides a schematic illustration of five alternatives.

In one approach, Studer and coworkers ${ }^{37}$ expanded committed mesencephalic dopamine neuron precursors obtained in rat embryos in culture. On removal of the mitogen bFGF, part of the cells differentiated into THpositive, presumed dopaminergic neurons. The expanded cells survived transplantation to the rat striatum, but the survival of the grafted TH-positive cells was poor. In recent studies, Yan, et al., ${ }^{40}$ have reported that the presence of ascorbic acid promotes dopaminergic differentiation when the mesencephalic precursors are proliferated or passaged for extended periods in vitro. Additionally, Studer, et al., ${ }^{36}$ found that when the predifferentiation of the precursors was conducted in cultures with low oxygen, both proliferation and dopaminergic differentiation were 
enhanced. It remains unknown, however, whether ascorbic acid and low oxygen will increase the yield of surviving dopaminergic neurons after transplantation in vivo.

In the second approach, Carvey and collaborators $6,27,32$ expanded mesencephalic progenitors from rat embryos under epidermal growth factor stimulation in neurosphere cultures. The cells could subsequently be differentiated into a dopaminergic phenotype in response to signals provided by a combination of cytokines, glial cell line-derived neurotrophic factor, mesencephalic membrane fragments, and striatum-conditioned medium. The generated cells survived transplantation to the rat striatum but the survival was clearly lower than that in grafts of primary embryonic mesencephalic dopamine neurons.

In the third approach, Wagner and coworkers ${ }^{38}$ induced a dopaminergic phenotype in an immortalized multipotent neural stem cell line by overexpression of Nurr-1, in combination with as yet unidentified factors derived from Type 1 astrocytes of ventral mesencephalic origin. Nurr-1 is a transcription factor that is likely to play a critical role in development of mesencephalic dopamine neurons. Most of the Nurr-1-transduced cells expressed the THpositive enzyme as well as two other markers of mesencephalic dopamine neurons. The engineered neurons survived transplantation to the mouse striatum, but the yield was very low.

In the fourth approach, dopamine and serotonin neurons were generated in high yield from mouse embryonic stem cells in vitro. ${ }^{21}$ The undifferentiated stem cells were expanded, and central nervous system stem cells were selected and then expanded in the presence of bFGF. The cells were then differentiated to TH-positive neurons by removal of the mitogen. Transplantation of these cells was not performed. An important step forward in the generation of dopamine neurons from embryonic stem cells was recently reported by the same group. ${ }^{17}$ In their study they overexpressed the transcription factor, Nurr-1, in mouse embryonic stem cells, which were then differentiated to dopamine neurons in culture. The overexpression with Nurr-1 dramatically increased the yield of cells with a dopaminergic phenotype - that is, expressing dopaminergic markers, exhibiting dopamine release in vitro, and showing electrophysiological properties of dopamine neurons. When the cells were grafted to the rat striatum, they survived, extended processes, and improved deficits resembling symptoms exhibited in PD patients. We do not, however, yet know if the cells induce functional reinnervation of the striatum, or what their symptomatic efficacy (for example, compared with primary embryonic neurons) and long-term safety is. Furthermore, it remains to be determined whether this type of genetic modification of the embryonic stem cells is acceptable in a clinical setting.

In a fifth approach, Kawasaki and collaborators ${ }^{16}$ studied mouse embryonic stem cells. They cocultured the embryonic stem cells with various cell lines and discovered that a bone marrow-derived stromal cell line was a potent inducer of neuronal differentiation. After coculture, almost all cultures contained differentiated neurons and there was a significant yield of TH-positive neurons. These cells produced dopamine and showed substantial short-term survival (at 2 weeks) after transplantation to the mouse striatum.

Although these data are promising and support the no- tion that it will become possible to generate dopamine neurons from stem cells for transplantation purposes, several unresolved issues remain. One problem is that the survival of these predifferentiated dopamine neurons after transplantation in animal models, when this has been tested, has been poor in most cases. Virtually nothing is known about long-term survival. It is also unclear if these cells display the functional characteristics of fully mature mesencephalic dopamine neurons after grafting, which is probably crucial if they should work in a clinical setting. Additionally, relatively little is known about human cells because the authors of most studies have used cells obtained in rodents.

The finding that the adult human brain also contains neural stem cells has raised the possibility that the patient's own neural stem cells could be used to generate, for example, dopamine neurons for transplantation. The cells would then be removed, predifferentiated in vitro, and reimplanted. One major advantage could be the lack of any immune reaction. Several problems with this approach, however, might occur. First, it will probably involve additional surgery in patients with an already diseased brain. Second, it is not known if these human cells can be expanded in sufficient numbers and if they can be differentiated into specific neurons such as dopamine neurons. Finally, in patients with a chronic neurodegenerative disorder, these cells may be functionally impaired because of age, disease process, or exposure to long-term drug treatment.

\section{CONCLUSIONS}

The most important scientific conclusion to be drawn from the clinical trials involving neural transplantation in PD is that cell replacement can be effective in the diseased human brain. It is important, however, to underscore that a clinically useful cell therapy for PD does not yet exist. There are several problems linked to the use of primary embryonic tissue in current procedures. First, there is a paucity of sufficient amounts of tissue for transplantation in a large number of patients. Second, posttransplantation functional outcome varies, with major improvement in some patients and only modest if any clinical benefit in others. This lack of consistent efficacious results is probably due to several factors such as variation in the viability and composition of the graft tissue but also issues related to patient selection and optimal graft placement. Third, although most likely not due to overgrowth of the dopaminergic graft, it is clear that dyskinesias can occur after implantation of human embryonic tissue. We must understand the underlying mechanisms and be able to avoid this adverse effect.

To conclude, neural transplantation is still at an experimental stage in PD. Several scientific problems must be addressed before this approach should be further tested in patients. With the development of new effective treatments for patients with advanced PD, such as deep brain stimulation, it is necessary to ask whether it is justified to make any further efforts to develop cell-based therapies for this disorder. In our opinion, cell therapy, if successful, offers several unique features and distinct advantages over other treatment strategies. The aim of this therapy is to restore dopamine transmission in the striatum-that is, in 
the precise area that exhibits the most pronounced loss of intrinsic dopamine innervation. In successful cases, this has given rise to major clinical improvements and allowed for withdrawal of antiparkinsonian medication, in the absence of major side effects. Furthermore, the grafted neurons are not destroyed by the disease process in cases followed at least 10 years after surgery, indicating that the symptomatic relief can be maintained for many years.

Currently, dopamine neurons generated from stem cells seem to be the most promising alternative to the use of primary human embryonic tissue for grafting in PD. We need, however, to learn much more about the mechanisms of dopamine cell differentiation, regeneration, and functional recovery. We are uncertain of the best stem cell source for generating new dopamine neurons. Thus, the development of stem cell-based therapies for PD remains in its infancy and it is crucial that scientists and clinicians progress with great care.

\section{References}

1. Annett LE: Functional studies of neural grafts in parkinsonian primates, in Dunnett SB, Björklund A (eds): Functional Neural Transplantation. New York: Raven Press, 1994, Vol 2, pp 71-102

2. Björklund LM, Sánchez-Pernaute R, Chung S, et al: Embryonic stem cells develop into functional dopaminergic neurons after transplantation in a Parkinson rat model. Proc Natl Acad Sci USA 99:2344-2349, 2002

3. Brundin P, Duan WM, Sauer H: Functional effects of mesencephalic dopamine neurons and adrenal chromaffin cells grafted to the rodent striatum, in Dunnett SB, Björklund A (eds): Functional Neural Transplantation. New York: Raven Press, 1994, Vol 2, pp 9-46

4. Brundin P, Karlsson J, Emgård M, et al: Improving the survival of grafted dopaminergic neurons: a review over current approaches. Cell Transplant 9:179-195, 2000

5. Brundin P, Pogarell O, Hagell P, et al: Bilateral caudate and putamen grafts of embryonic mesencephalic tissue treated with lazaroids in Parkinson's disease. Brain 123:1380-1390, 2000

6. Carvey PM, Ling ZD, Sortwell CE, et al: A clonal line of mesencephalic progenitor cells converted to dopamine neurons by hematopoietic cytokines: a source of cells for transplantation in Parkinson's disease. Exp Neurol 171:98-108, 2001

7. Deacon T, Schumacher J, Dinsmore J, et al: Histological evidence of fetal pig neural cell survival after transplantation into a patient with Parkinson's disease. Nat Med 3:350-353, 1997

8. Defer GL, Geny C, Ricolfi F, et al: Long-term outcome of unilaterally transplanted parkinsonian patients. I. Clinical approach. Brain 119:41-50, 1996

9. Fahn S, Elton RL, Members of the UPDRS Development Committee. Unified Parkinson's Disease Rating Scale, in Fahn S, Marsden CD, Calne DB, et al (eds): Recent Developments in Parkinson's Disease. Florham Park: MacMillan Healthcare Information, 1987, Vol 2, pp 153-163

10. Freed CR, Greene PE, Breeze RE, et al: Transplantation of embryonic dopamine neurons for severe Parkinson's disease. $\mathbf{N}$ Engl J Med 344:710-719, 2001

11. Freeman TB, Olanow CW, Hauser RA, et al: Bilateral fetal nigral transplantation into the postcommissural putamen in Parkinson's disease. Ann Neurol 38:379-388, 1995

12. Hagell P, Brundin P: Cell survival and clinical outcome following intrastriatal transplantation in Parkinson disease. J Neuropathol Exp Neurol 60:741-752, 2001

13. Hagell P, Piccini P, Björklund A, et al: Dyskinesias following neural transplantation in Parkinson's disease. Nat Neurosci 5: $627-628,2002$
14. Hagell P, Schrag A, Piccini P, et al: Sequential bilateral transplantation in Parkinson's disease: effects of the second graft. Brain 122:1121-1132, 1999

15. Hauser RA, Freeman TB, Snow BJ, et al: Long-term evaluation of bilateral fetal nigral transplantation in Parkinson disease. Arch Neurol 56:179-187, 1999

16. Kawasaki H, Mizuseki K, Nishikawa S, et al: Induction of midbrain dopaminergic neurons from ES cells by stromal cell-derived inducing activity. Neuron 28:31-40, 2000

17. Kim JH, Auerbach JM, Rodriguez-Gomez JA, et al: Dopamine neurons derived from embryonic stem cells function in an animal model of Parkinson's disease. Nature 418:50-56, 2002

18. Kordower JH, Freeman TB, Chen EY, et al: Fetal nigral grafts survive and mediate clinical benefit in a patient with Parkinson's disease. Mov Disord 13:383-393, 1998

19. Kordower JH, Freeman TB, Snow BJ, et al: Neuropathological evidence of graft survival and striatal reinnervation after the transplantation of fetal mesencephalic tissue in a patient with Parkinson's disease. New Engl J Med 332:1118-1124, 1995

20. Kordower JH, Rosenstein JM, Collier TJ, et al: Functional fetal nigral grafts in a patient with Parkinson's disease: chemoanatomic, ultrastructural, and metabolic studies. J Comp Neurol 370:203-230, 1996

21. Lee SH, Lumelsky N, Studer L, et al: Efficient generation of midbrain and hindbrain neurons from mouse embryonic stem cells. Nat Biotechnol 18:675-679, 2000

22. Lindvall O: Rationales and strategies of fetal neural transplantation in Parkinson's disease, in Krauss JK, Jancovic J, Grossman RG (eds): Surgery for Parkinson's Disease and Movement Disorders. Philadelphia: Lippincott Williams \& Wilkins, 2001, pp 194-209

23. Lindvall $\mathrm{O}$, Brundin $\mathrm{P}$, Widner $\mathrm{H}$, et al: Grafts of fetal dopamine neurons survive and improve motor function in Parkinson's disease. Science 247:574-577, 1990

24. Lindvall O, Hagell P: Clinical observations after neural transplantation in Parkinson's disease. Prog Brain Res 127: 299-320, 2000

25. Lindvall O, Sawle G, Widner H, et al: Evidence for long-term survival and function of dopaminergic grafts in progressive Parkinson's disease. Ann Neurol 35:172-180, 1994

26. Lindvall $\mathrm{O}$, Widner $\mathrm{H}$, Rehncrona $\mathrm{S}$, et al: Transplantation of fetal dopamine neurons in Parkinson's disease: one-year clinical and neurophysiological observations in two patients with putaminal implants. Ann Neurol 31:155-165, 1992

27. Ling Z, Potter ED, Lipton JW, et al: Differentiation of mesencephalic progenitor cells into dopaminergic neurons by cytokines. Exp Neurol 149:411-423, 1998

28. Mendez I, Dagher A, Hong M, et al: Enhancement of survival of stored dopaminergic cells and promotion of graft survival by exposure of human fetal nigral tissue to glial cell line-derived neurotrophic factor in patients with Parkinson's disease. Report of two cases and technical considerations. J Neurosurg 92: 863-869, 2000

29. Peschanski M, Defer G, N'Guyen JP, et al: Bilateral motor improvement and alteration of L-dopa effect in two patients with Parkinson's disease following intrastriatal transplantation of foetal ventral mesencephalon. Brain 117:487-499, 1994

30. Piccini P, Brooks DJ, Björklund A, et al: Dopamine release from nigral transplants visualized in vivo in a Parkinson's patient. Nat Neurosci 2:1137-1140, 1999

31. Piccini P, Lindvall O, Björklund A, et al: Delayed recovery of movement-related cortical function in Parkinson's disease after striatal dopaminergic grafts. Ann Neurol 48:689-695, 2000

32. Potter ED, Ling Z, Carvey PM: Cytokine-induced conversion of mesencephalic-derived progenitor cells into dopamine neurons. Cell Tissue Res 296:235-246, 1999

33. Remy P, Samson Y, Hantraye P, et al: Clinical correlates of $\left[{ }^{18} \mathrm{~F}\right]$ fluorodopa uptake in five grafted parkinsonian patients. Ann Neurol 38:580-588, 1995 


\section{Cell therapy in Parkinson disease}

34. Sawle GV, Bloomfield PM, Björklund A, et al: Transplantation of fetal dopamine neurons in Parkinson's disease: PET $\left[{ }^{18} \mathrm{~F}\right] 6-1-$ fluorodopa studies in two patients with putaminal implants. Ann Neurol 31:166-173, 1992

35. Schumacher JM, Ellias SA, Palmer EP, et al: Transplantation of embryonic porcine mesencephalic tissue in patients with PD. Neurology 54:1042-1050, 2000

36. Studer L, Csete M, Lee SH, et al: Enhanced proliferation, survival, and dopaminergic differentiation of CNS precursors in lowered oxygen. J Neurosci 20:7377-7383, 2000

37. Studer L, Tabar V, McKay RD: Transplantation of expanded mesencephalic precursors leads to recovery in parkinsonian rats. Nat Neurosci 1:290-295, 1998

38. Wagner J, Åkerud P, Castro DS, et al: Induction of a midbrain dopaminergic phenotype in Nurr1-overexpressing neural stem cells by type 1 astrocytes. Nat Biotechnol 17:653-659, 1999

39. Wenning GK, Odin P, Morrish P, et al: Short-and long-term survival and function of unilateral intrastriatal dopaminergic grafts in Parkinson's disease. Ann Neurol 42:95-107, 1997
40. Yan J, Studer L, McKay RD: Ascorbic acid increases the yield of dopaminergic neurons derived from basic fibroblast growth factor expanded mesencephalic precursors. J Neurochem 76:307-311, 2001

Manuscript received September 23, 2003.

Accepted in final form October 14, 2003.

This work was supported by grants from the Swedish Research Council, the Kock Foundation, the Wiberg Foundation, the King Gustav V and Queen Victoria Foundation, and the Söderberg Foundation.

Address reprint requests to: Olle Lindvall, M.D., Ph.D., Section of Restorative Neurology, Wallenberg Neuroscience Center/BMC A11, University Hospital, SE-221 84 Lund, Sweden. email: olle. lindvall@neurol.lu.se. 